\title{
Simulation and Optimization of Furrow Irrigation by the Model SASIS: Sensibility to the Spatial and Temporal Weighted Factors
}

\author{
Roberto Vieira Pordeus ${ }^{1}$, Carlos Alberto Vieira de Azevedo ${ }^{2}$, Valeria Ingrith Almeida Lima ${ }^{1}$, Silvanete Severino \\ da Silva ${ }^{1}$, Vera Lúcia Antunes de Lima ${ }^{2}$ and Márcia Rejane de Queiroz Almeida Azevedo ${ }^{3}$ \\ 1. Department of Environmental Science and Technology, Rural Federal University of Semiarid, Mossor, RN 59600-000, Brazil \\ 2. Department of Agricultural Engineering, Federal University of Campina Grande, Campina Grande, PB 58429-170, Brazil \\ 3. Department of Agroecology, University of Paraíba State, Lagoa Seca, PB 58117-000, Brazil
}

Received: July 29, 2011 / Accepted: November 2, 2011 / Published: April 20, 2012.

\begin{abstract}
The surface irrigation systems are the most used not just in Brazil, but in the whole world mainly due to the economy of energy and its operation easiness; however, these systems present low levels of performance, usually, as a consequence of inadequate design and management. Thus, the objective of this research was to develop a tool capable to make possible the simulation and optimization of the continuous flow furrow irrigation performance, making successive simulations of the advance phase and respective prognostics of the performance parameters of the irrigation system. The proposed model is denominated SASIS, "Software Applied to Simulation of the Surface Irrigation”, and had its validation tested for different field conditions. In this paper, the simulation and the optimization of the furrow irrigation by the model SASIS are evaluated regarding to the spatial $(\phi)$ and temporal $(\theta)$ weighted factors, which account for the nonlinear variation in the surface and subsurface flow profiles. The analysis of the sensitivity of simulation of the advance phase in the irrigation identified ranges of combinations between spatial and temporal weighted factors of the surface and subsurface flow profiles, resulted in acceptable discrepancies between simulated and measured advance time. Also it was verified that conjugated effect of these weighted factors on the simulation of the advance phase was greater than the effect of the temporal weighted factor. The model presents effective mechanisms in the accomplishment of countless simulations, in a discharge strip understood between the minimum and the maximum allowable values, aiming to determine the relationship between discharge and water application efficiency, deep percolation and runoff rates, and consequently to optimize the performance of the furrow irrigation systems with continuous flow.
\end{abstract}

Key words: Furrow irrigation, simulation, optimization.

\section{Introduction}

The method of surface irrigation is considered the oldest form of artificial application of water in the soil in order to meet the water requirements of crops; it is the most widely used method of the world, especially in the Asian and African continents, and even in the USA and in the most developed countries of Europe. In Brazil, despite the lack of accuracy, it covers the

Corresponding author: Roberto Vieira Pordeus, Ph.D., main research fields: irrigation and drainage. E-mail: rpordeus@ufersa.edu.br. largest irrigated area because, undoubtedly, of its pioneering during the deployment of large public irrigation projects, particularly in the northeast.

Although the surface irrigation is the most widely used one of the world, it is considered low efficiency in water application, especially the furrow irrigation system, in which the ones of open furrow are responsible for the lower levels of efficiency. The low efficiency in the surface irrigation systems is due mainly to the lack of a careful design and to the practice of improper management of irrigation. 
According to Rezende et al. [1], reduced levels of performance in furrow irrigation systems can be attributed to the incorrect design as well as to the unsatisfactory operation and management.

The furrow irrigation presents different field and operating variables of the system which influence its performance, like flow and time of water application, dimensions, slope and roughness of the surface soil, geometry of the furrow and caracteristics of water infiltration in the soil; the values of variables like slope, roughness, geometry of the furrow and average of infiltration correspond to specific conditions of field, for which the designer must define flow, time of water application, length, row spacing and water depth. A good furrow irrigation project must consider these variables and the interaction among them [2, 3].

The surface irrigation systems present the potential of applying water to crops with efficiency of $70 \%$ to $80 \%$ [4]; actually, some automated systems have favored efficiency about 90\% [5]; but most irrigation projects of the world have worked with efficiency of application about $40 \%$ to $50 \%$ or with values even lower [5-6].

The surface irrigation process takes place in four phases: advance, storage, depletion and recession, in which the first one starts when the water is adduced and ends when the advancing front reaches the end of the furrow or covers the entire portion of a range or board, starting the storage phase, in which water level in the surface soil rises, ending when the flow is canceled. At that time, the depletion phase starts and ends when the water disappears in any portion along the furrow or range, what generally happens in the headboard. Then, it happens the recession phase, when water level starts demoting, and which ends when all the water disappeares on the surface soil. In furrow irrigation, Strelkoff et al. [7] considered the storage and depletion as only one phase, i.e. the event of furrow irrigation is divided into three phases: advance, storage/depletion and recession.

According to Strelkoff et al. [7], the acompaniment of the various phases of surface irrigation permits the determination of the advance and recession curves; for such a thing, it is necessary to go to the field in order to measure the time in which water advances to specific predetermined points, $x_{r}$, and the time of its disappearance in the same points, $t_{r}$. In graph paper, the points are plotted $\left(x_{r}, t_{r}\right)$ determining the advance and recession curves. Another possibility is the mathematical simulation of the phases which, somewhat, reduces the requirements for field measurements, which consume time and money [8]. According to Strelkoff et al. [7], the knowledge about the advance and recession curves and the characteristic equation of soil infiltration is essential to evaluate the performance of a surface irrigation system, concerning to the determination of parameters which express the quality of the irrigation, especially the application efficiency $(E a)$, storage $(E s)$, distribution $(E d)$ and the percolation (Ip) and runoff (Iro) rates.

According to Refs. [8, 9], the low application efficiency of the surface irrigation projects is due to an inappropriate design and management, which become complicated because of the spatial and temporal variation of the field parameters, especially the ones of water infiltration in soil. According to Azevedo et al. [9], the irrigation projects in Brazil are, in general, operated with low efficiency because the perspective used is the one in which the irrigation systems must be well designed and constructed but, after the deployment, the management system is not considered, becoming, with rare exceptions, exclusively dependent on the sensitivity and experience of the farmer.

The different simulation models of surface irrigation were developed in order to simulate an isolated irrigation event, assuming that there is not spatial variability in the field parameters (infiltration, roughness, slope and cross-sectional area); in practice, the validity of this hypothesis has been verified considering that the simulations are very close to the field measurements of the phases, however, the time 
variability in these parameters are always taken into account, as for the evaluation of any irrigation event throughout the growing season, new measurements of field parameters are held.

With this research, it aimed to develop a mathematical computational model for simulation and optimization of furrow irrigation with continuous flow and, through the simulation of the advance phase, able to predict the performance of an irrigation event and select the optimum flow in the furrow irrigation with continuous flow, i.e. which maximizes the water application efficiency, balancing losses by percolation and runoff.

\section{Material and Methods}

In the kinematic wave model used in this research, it is assumed that there is not variation of the height of flow with the distance, i.e., $\partial y / \partial x=0$ neglecting completely the equation of motion, without what nothing can be said concerning to the dynamics of the shape of surface profile of flow, getting the equation of continuity indefinite in time $\partial A / \partial t$. To solve this problem, it is assumed the existence of a unique relationship which describes flow as function of the flow area. Then, it is substituted the equation of motion by the Manning Equation. The runoff, studied only cinematically, is similar to the propagation of a kinematic wave which collides, why these models are called kinematic waves. The designation of uniform runoff models also used is due to, obviously, the fundaments of the equation of uniform runoff.

Because this kind of model is not applicable to furrows when the slope is too small, i.e., when the slope tends to zero: actually its precision will decrease when $S_{o}$ approximates to zero [10].

Therefore, the equations of the kinematic waves model used become:

Continuity equation

$$
\begin{gathered}
\frac{\partial A}{\partial t}+\frac{\partial Q}{\partial x}+\frac{\partial Z}{\partial \tau}=0 \\
Q=\alpha A^{m}
\end{gathered}
$$

(Manning Equation) where

$$
\begin{array}{r}
\alpha=\frac{\sqrt{\rho_{1} S_{O}}}{n} \\
m=\frac{\rho_{2}}{2}
\end{array}
$$

where:

$A=$ cross-sectional of flow area, $\mathrm{m}^{2}$;

$t=$ time of occurence, second;

$x=$ distance of water advance in field, $\mathrm{m}$;

$\tau=$ time of infiltration opportunity, second;

$Z$ = infiltrated volume accumulated per unit length of furrow, $\mathrm{m}^{3} \cdot \mathrm{m}^{-1}$;

$Q=$ discharge flow, $\mathrm{m}^{3} \cdot \mathrm{s}^{-1}$;

$n=$ Manning roughness coefficient, $\mathrm{m}^{-1 / 3} \cdot \mathrm{s}$;

$S_{o}=$ slope of field, $\mathrm{m} \cdot \mathrm{m}^{-1}$;

$\rho_{1}$ and $\rho_{2}=$ empirical constants adjusted to the field measurements of the geometry of the furrow;

$\alpha$ and $m=$ empirical constants.

For spatial numerical solution of the equations of the kinematic waves model the Eulerian integration procedure with first order approximation by Refs. [2, $11]$ was used, in this research, which resulted in two algebraic equations more stable and easier to be dissolved in microcomputers. Conceptually, the approximation considers the surface and subsurface profiles of water throughout the wetted area during sequential stages of calculation. Fig. 1 illustrates the surface and subsurface profiles of flow in the times $t_{i-1}$ and $t_{i}$, identifying the cells which compose them. During each stage of calculation, the water flow advances an incremental distance, $\delta x$; e.g., during the first interval (first stage of calculation), the water advance extends to a distance $\delta x_{1}$; in the second interval, to a distance $\delta x_{2}$, and so on. It can be generalized to the distance of the advancing front, $x_{i}$, in the time $t_{i}$, as it follows:

$$
x_{i}=\sum_{k=1}^{i} \delta x_{k}
$$

where, $\delta x_{k}=k^{\text {th }}$ increment of space, defined by the advance during the interval, when $i=k$, where $k$ is the number of time increment. 

Temporal Weighted Factors

A typical cell of the profile is illustrated in Fig. 2, displaying profiles in the stages of calculation $t_{i-1}$ and $t_{i}$. Notes $J, M, L$ and $R$ are introduced in each cell in order to identify the variables which describe flow conditions related to time and space. Thus, the variables subscripted by $J$ or $M$ reffer to the flow conditions in time $t_{i-1}$ and borders left (upstream) and right (downstream) of the cell, respectively. Similarly, $\mathrm{L}$ and $\mathrm{R}$ are subscripted borders left and right of the cell in time $t_{i}$. Combining the cells of all time incremets there will be a grid calculation in the line $(x$, $t$ ), in which the advance and recession trajectories may be drawn. It is observed, in this line, that during the advance phase the cells are rectangular, except the ones of the advancing front, which are triangular due not existing flow in the downstream borders of these cells at the times $t_{i}$ and $t_{i-1}$. During the storage and depletion phases, all cells are rectangular; and during the recession, the cell end upstream is triangular for the same reasons of flow behavior in the advancing front, but the other cells are rectangular. The width of each cell is determined by the distance of the advancing front during each stage of calculation $\delta t$, which becomes, then, one of the unknowns of the problem, once it was considered $\delta$ t constant. This is called a spatial solution of the Saint-Venant equations because $\delta x$ is an unknown, while the value of $\delta t$ is defined by the user model.

The integrated form of the equation of continuity in relation to distance and time results in:

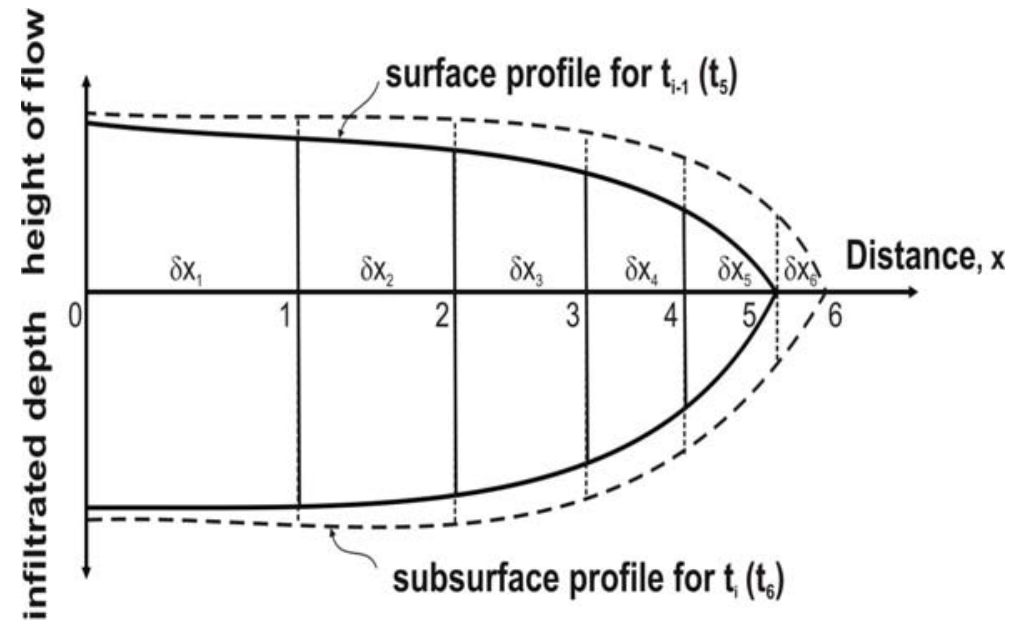

Fig. 1 Schema of progression of superficial flow and infiltration for the constant interval. Source: Ref. [11].

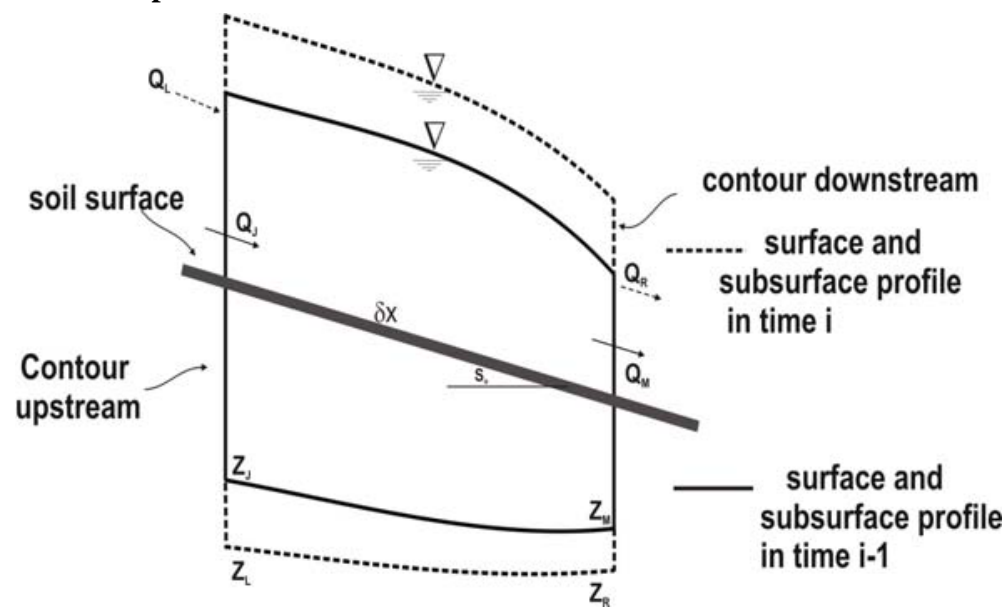

Fig. 2 Deformed cell of flow [11]. 


$$
\begin{aligned}
& \frac{1}{2}\left[\left(Q_{x+\delta x, t}-Q_{x, t}\right)_{t+\delta t}+\left(Q_{x+\delta x, t}-Q_{x, t}\right)_{t}\right] \delta t+ \\
& \frac{1}{2}\left[\left(A_{x, t+\delta t}-A_{x, t}\right)_{x+\delta x}+\left(A_{x, t+\delta t}-A_{x, t}\right)_{x}\right] \delta x+ \\
& \frac{1}{2}\left[\left(Z_{x, t+\delta t}-Z_{x, t}\right)_{x+\delta x}+\left(Z_{x, t+\delta t}-Z_{x, t}\right)_{x}\right] \delta x=0
\end{aligned}
$$

Since during the advance phase the flow decreases throughout the distance for the effect of the rate of water infiltration in soil, falling to zero in the advancing front, the non-linearity of the profile, both surface and subsurface, becomes quite marked, making the factor $1 / 2$ not appropriated to consider the flow conditions; thus, an appropriate weighted must be from $1 / 2$ to 1 . Then, the factors $\theta$ and $\phi$ are created to consider the non-linearity of the profile, respectively, related to time and space. These factors are extremely important only during the advance phase because after this phase there is tendency to establish stable conditions of flow. The weighted must be superior in time $t+\delta t$ as during the advance phase the non-linearity increases with time, getting maximum when the water reaches the end of the area (zero flow conditions); besides, also there must be superior in the input section because the height of flow varies soon in this section, since it is in this section where flow is being applied.

Replacing, in Eq. (6), 1/2 by $\theta,(1-\theta), \phi,(1-\phi)$ and writing the flow variables in terms of the notes of a computational cell, there is:

Eq. (6) may, then, be represented in terms of the notes, in Fig. 2, as follows:

$$
\begin{aligned}
& {\left[\theta\left(Q_{R}-Q_{L}\right)+(1-\theta)\left(Q_{M}-Q_{J}\right)\right] \delta t+} \\
& {\left[\phi\left(A_{L}-A_{J}\right)+(1-\phi)\left(A_{R}-A_{M}\right)\right] \delta x+} \\
& {\left[\phi\left(Z_{L}-Z_{J}\right)+(1-\phi)\left(Z_{R}-Z_{M}\right)\right] \delta x=0}
\end{aligned}
$$

where, $\theta$ and $\phi$ are temporal and spatial weighted factors, respectively, whose values vary from $1 / 2$ and 1 ; in general, it has taken values equal to 0.65 and 0.51 for $\theta$ and $\phi$, respectively.

The numerical solution is obtained by solving Eq. (7) for each cell in the computational mesh starting horizontally from left to right, in each stage of calculation. The only unknowns in each cell are $Q_{R}$ and $A_{R}$; however, once $Q$ is explicitly being calculated on Manning equation (Eq. (2)), it is not considered an unknown; the same case is applied to $Z$ (infiltration) which is being calculated on Kostiakov-Lewis equation. What is done is to replace the equations of $Q$ and $Z$ by Eq. (7) and, then, it gets only one unknown $\left(A_{R}\right)$.

Replacing Eq. (2) by Eq. (7) and dividing it by $\theta \alpha / \delta t$, there will be the following equation nonlinear in $A_{R}$ :

$$
\begin{aligned}
& A_{R}^{m}+\left(\frac{1-\phi}{\theta \alpha}\right) \frac{\delta x}{\delta t} A_{R}-A_{L}^{m}+\left(\frac{1-\theta}{\theta}\right)\left(A_{M}^{m}-A_{J}^{m}\right)+ \\
& \frac{\phi}{\theta \alpha}\left(A_{L}+Z_{L}-A_{J}-Z_{J}\right) \frac{\delta x}{\delta t}+\left(\frac{1-\phi}{\theta \alpha}\right)\left(Z_{R}-A_{M}-Z_{M}\right) \frac{\delta x}{\delta t}=0
\end{aligned}
$$

Aiming to simplify Eq. (8), the constants and variables with values known from the previous stage of calculation in the coefficients $C_{1}$ and $C_{2}$ are isolated. Then, there is:

$$
C_{1}=\left(\frac{1-\phi}{\theta \alpha}\right) \frac{\delta x}{\delta t}
$$

and

$$
\begin{aligned}
& C_{2}=-A_{L}^{m}-\left(\frac{1-\theta}{\theta}\right) A_{J}^{m+1}+\left(\frac{1-\theta}{\theta}\right) A_{M}^{m+1}+ \\
& \frac{\phi}{\alpha \theta}\left(A_{L}+Z_{L}-A_{J}-Z_{J}\right) \frac{\delta x}{\delta t}+\left(\frac{1-\phi}{\alpha \theta}\right)\left(Z_{R}-A_{M}-Z_{M}\right) \frac{\delta x}{\delta t}
\end{aligned}
$$

getting the equation

$$
A_{R}^{m+1}+C_{1} A_{R}+C_{2}=0
$$

Eq. (11) is used for interior cells and for the first one after the first stage of calculation. In this case a matrix does not exist because the Eq. (11) is solved implicitly (by Newton-Raphson's method) for each cell, separately. This equation is used implicitly to determine $A_{R}$ and, after, it is determined explicitly $Q_{R}$, by Manning equation (Eq. (2)).

Equation of water infiltration was obtained on equation:

$$
Z=k \tau^{a}+f_{o} \tau
$$

where:

$Z=$ accumulated infiltration, $\mathrm{m}^{3} \cdot \mathrm{m}^{-1} \cdot \mathrm{min}^{-1}$;

$\tau$ = time of infiltration opportunity, min; 
$k=$ constant of Kostiakov-Lewis equation of infiltration, $\mathrm{m}^{3} \cdot \mathrm{min}^{-\mathrm{a}} \cdot \mathrm{m}^{-1}$;

$a=$ empirical coefficients of Kostiakov-Lewis equation of infiltration;

$f_{o}=$ basic infiltration rate in $\mathrm{m}^{3} \cdot \mathrm{m}^{-1} \cdot \mathrm{min}^{-1}$.

The maximum nonerosive flow was obtained through Eq. (13):

$$
Q_{\max }=\left[\left(\frac{V_{\max } \rho_{2} n^{2}}{3600 \quad S_{0} \rho_{1}}\right)\right]^{\frac{1}{\rho_{2}-2}}
$$

where

$Q_{\text {max }}=$ maximun nonerosive flow, $\mathrm{m}^{3} \cdot \mathrm{min}^{-1}$;

$V_{\text {max }}=$ maximun nonerosive velocity, $\mathrm{m} \cdot \mathrm{min}^{-1}$;

$n=$ Manning coefficient, $\mathrm{m}^{-1 / 3} \cdot \mathrm{s}$;

$\rho_{1}$ and $\rho_{2}=$ coefficients which express the geometry of the furrow, dimensionless;

$S_{o}=$ slope of the furrow, $\mathrm{m} \cdot \mathrm{m}^{-1}$.

The infiltrated volume was determined by using the trapezoidal rule by Eq. (14):

$$
V_{z}=\frac{L}{2 n}\left[Z_{o}+\left(2 Z_{1}+2 Z_{2}+\ldots+2 Z_{n-1}\right)+Z_{n}\right]
$$

where:

$L=$ length of the area;

$Z_{i}=$ accumulated infiltration to point $i, \mathrm{~m}^{3} \cdot \mathrm{m}^{-1}$;

$n=$ number of segments in which the furrow is subdivided.

The accumulated infiltration in each segment of the furrow is given by:

$$
Z_{i}=k\left[t_{r}-\left(t_{a}\right)_{i}\right]^{a}+f_{o}\left[t_{r}-\left(t_{a}\right)_{i}\right]
$$

where:

$k=$ Kostiakov-Lewis equation constants, $\mathrm{m}^{3} \cdot \mathrm{min}^{-\mathrm{a}} \cdot \mathrm{m}^{-1}$;

$a=$ Kostiakov-Lewis equation empirical constants;

$\mathrm{f}_{\mathrm{o}}=$ basic infiltration rate, $\mathrm{m}^{3} \cdot \mathrm{m}^{-1} \cdot \mathrm{min}^{-1}$;

$t_{r}=$ time of recession, min;

$\left(t_{a}\right)_{i}=$ time of advance for ai ${ }^{\text {th }}$ station, min.

The recession phase is marked by disappearance of water from surface soil. According to some authors, the recession occurs as soon as the water application ends. In this work, the depletion and recession phases were neglected, considering that the cutting time, $t_{\text {com }}$, replaces $t_{r}$ in Eq. (15).

\section{Results and Discussion}

Certain variables introduced in the model SASIS influenced the results of the simulation of the furrow irrigation with continuous flow. The effect of some variables is isolated while other ones have a conjugated effect, i.e., the effect of one depends on the effect of the other. Later, the fact of having, perhaps, a combined effect between the spatial $(\phi)$ and temporal $(\theta)$ weighted factors will be highlighted. An appropriate choice of the weighted factors of the integration of the deformable cells advance contributes to a better simulation of the advancing curve, favoring a more precise prognosis of the performance of irrigation. In order to evaluate the simulation of the software in relation to the spatial $(\phi)$ and temporal $(\theta)$ weighted factors, it used the AMALGACQ field data, collected by Azevedo [9], having fixed all the other parameters and varied the spatial weighted factor $(\phi)$, in the interval from 0.51 to 0.71 , and the temporal weighted factor $(\theta)$, in the interval from 0.51 to 0.61 . In Table 1 , it is observed that for set of values of $\phi$ and $\theta$ of ( 0.53 and $0.57 ; 0.57$ and $0.59 ; 0.59$ and $0.59 ; 0.65$ and $0.61 ; 0.60$ and 0.60 ) occurred variations in the advance time which varied from $14 \%$ to $22 \%$ in relation to the average advance time in field. Therefore, it is noticed the existance of various combinations of values of these weighted factors which are able to simulate the advance phase with acceptable variations in the prognosis of the advance time. It is also verified ranges of combinations of these weighted factors which do not permit simulations of the advance phase, what certainly is due to linearity of the advance curve in this field data (AMALGACQ). Concerning to water application efficiency, water storage efficiency and to infiltrated volume, it was found that they are directly affected by the spatial and temporal weighted factors, occurring variations in the water application efficiency of $10.4 \%$ and in the water storage efficiency of $12.7 \%$ and in the infiltrated volume of $5.89 \mathrm{~m}^{3}$; the lowest value (58.5\%) estimated for the water application 
efficiency was for combination of the spatial and temporal weighted factors of 0.51 and 0.57 respectively, while the highest value (58.9\%) was for combination 0.69 and 0.51 ; for the water storage efficiency the lowest value estimated (87.1\%) was for combination (0.51 and 0.57), while the maximum value (100\%) was obtained for several pairs of combinations; regarding the infiltrated volume, the highest value $\left(39.12 \mathrm{~m}^{3}\right)$ was estimated when the combination 0.69 and 0.51 was used for spatial and temporal weighted factors, respectively, while the lowest value estimated for the infiltrated volume (33.3 $\mathrm{m}^{3}$ ) was when it was used 0.51 for the spatial weighted factor $(\phi)$ and 0.57 for the temporal weighted factor $(\theta)$. Therefore, it was observed, somewhat, that the more rigorous in choosing the weighted factors $\phi$ and $\theta$ the better the result of the simulation. SIRMOD simulated, for these field data (AMALGACQ), advance time of $67.3 \mathrm{~min}$., estimating a water application efficiency and a water storage efficiency of 67.2 and $100 \%$ respectively, beyond an infiltrated volume of $41.43 \mathrm{~m}^{3}$ for the value of $\phi$ of 0.51 and $\theta$ of 0.60 . It is then concluded in Table 1 that there are several combinations of weighted factors in the Saint-Venant equation, which are able to improve the simulation of the advance phase with the model SASIS. It is recommended in the Software SASIS values close to 0.60 for both weighted factors. According to Carvalho et al. [12], spatial and temporal weighted factors, $\phi$ and $\theta$, are used in the integration, because of the nonlinearity of the continuity equation. Based on the values presented in Table 1, it is verified that there is a low variation in the water application efficiency, in the water storage efficiency and in the infiltrated volume with the increase of the weighted factors; the opposite is true with the advance time, i.e., it decreases whenever the weighted factors increase.

It is seen in Table 1 that the situations which best approximated to value measured in field were for $\phi$ and $\theta$ equal to 0.57 and 0.59 respectively, and $\phi$ and $\theta$ equal to 0.60 and 0.61 respectively; it is observed that the simulation is more affected when values of $\theta$ inferior to 0.55 and values of $\phi$ superior to 0.60 are considered, being simulation more affected by spatial weighted factor than by temporal weighted factor affirming, therefore, the values used for $\phi$ and $\theta(0.60$ and 0.60 ) by Carvalho et al. [12] in SIRMOD III and, here, by software SASIS.

Analyzing the effect of the spatial $\phi$, and temporal $\theta$, weighted factors on the water infiltrated volume, it is possible to observe that the water infiltrated volume

Table 1 Sensitivity analysis of simulation and optimization by model SASIS in relation to the variation of spatial ( $\phi)$ and temporal $(\theta)$ weighted factors, for the field data AMALGACQ (advance time = $232 \mathrm{~min})$.

\begin{tabular}{|c|c|c|c|c|c|c|c|c|c|c|c|c|c|c|c|c|c|c|c|c|c|c|c|c|}
\hline \multirow{2}{*}{$\theta / \phi$} & \multicolumn{4}{|c|}{0.51} & \multicolumn{4}{|c|}{0.53} & \multicolumn{4}{|c|}{0.55} & \multicolumn{4}{|c|}{0.57} & \multicolumn{4}{|c|}{0.59} & \multicolumn{4}{|c|}{0.61} \\
\hline & $T L$ & $E a$ & $E r$ & $V z$ & $T L$ & $E a$ & $E r$ & $V z$ & $T L$ & $E a$ & $E r$ & $V z$ & $T L$ & $E a$ & $E r$ & $V z$ & $T L$ & $E a$ & $E r$ & $V z$ & $T L$ & $E a$ & $E r$ & $V z$ \\
\hline 0.51 & 115 & 63.7 & 94.9 & 36.21 & 130 & 62.7 & 93.4 & 35.61 & 160 & 62.7 & 93.3 & 35.54 & 290 & 58.5 & 87.1 & 33.23 & & & & & & & & \\
\hline 0.53 & 110 & 63.4 & 94.3 & 36.04 & 125 & 63.8 & 95.0 & 36.20 & 145 & 62.2 & 92.6 & 35.28 & 195 & 60.4 & 89.0 & 34.28 & & & & & & & & \\
\hline 0.55 & 110 & 66.4 & 98.9 & 37.70 & 120 & 64.3 & 95.7 & 36.50 & 140 & 64.4 & 95.9 & 36.5 & 170 & 61.4 & 91.4 & 34.80 & & & & & & & & \\
\hline 0.57 & 105 & 65.2 & 97.0 & 37.06 & 115 & 64.2 & 95.6 & 36.49 & 130 & 63.5 & 94.5 & 36.0 & 155 & 62.1 & 92.4 & 35.19 & 265 & 58.9 & 87.7 & 33.42 & & & & \\
\hline 0.59 & 105 & 67.9 & 10 & 38.54 & 110 & 63.3 & 94.7 & 36.17 & 125 & 64.3 & 95.7 & 36.46 & 145 & 62.9 & 93.4 & 35.64 & 190 & 60.4 & 90.0 & 34.27 & & & & \\
\hline 0.60 & 100 & 64.6 & 96.2 & 36.78 & 110 & 65.0 & 96.8 & 36.94 & 120 & 62.9 & 93.7 & 35.74 & 140 & 62.8 & 93.5 & 35.62 & 180 & 61.5 & 91.6 & 34.87 & & & & \\
\hline 0.61 & 100 & 65.8 & 98.0 & 37.43 & 110 & 66.4 & 98.9 & 37.70 & 120 & 64.5 & 96.0 & 36.60 & 135 & 62.5 & 93.0 & 35.44 & 170 & 61.9 & 92.1 & 35.08 & & & & \\
\hline 0.63 & 100 & 68.1 & 10. & 38.70 & 105 & 64.9 & 96.7 & 36.91 & 115 & 64.2 & 95.5 & 36.44 & 130 & 63.5 & 94.6 & 36.03 & 155 & 62.2 & 92.7 & 35.29 & & & & \\
\hline 0.65 & 95 & 65.2 & 97.0 & 37.13 & 105 & 67.4 & 100 & 38.24 & 115 & 67.0 & 99.8 & 38.00 & 125 & 564.0 & 95.3 & 36.30 & 145 & 62.7 & 93.3 & 35.55 & 190 & 60.2 & 89.6 & 34.13 \\
\hline 0.67 & 95 & 67.5 & 99.8 & 38.16 & 100 & 65.0 & 96.8 & 36.99 & 110 & 65.7 & 97.9 & 37.24 & 120 & 63.9 & 95.2 & 36.28 & 140 & 64.2 & 95.6 & 36.37 & 170 & 61.5 & 91.6 & 34.88 \\
\hline 0.69 & 95 & 68.9 & 100 & 39.12 & 100 & 67.0 & 99.7 & 38.07 & 105 & 64.0 & 95.3 & 36.39 & 115 & 563.3 & 94.3 & 35.99 & 130 & 62.5 & 93.0 & 35.46 & 155 & 61.6 & 91.6 & 34.91 \\
\hline 0.71 & 90 & 64.7 & 96.3 & 36.93 & 100 & 68.9 & 100 & 39.10 & 105 & 66.1 & 98.4 & 37.53 & 115 & 565.7 & 97.8 & 37.26 & 125 & 62.9 & 93.7 & 35.72 & 150 & 63.3 & 94.3 & 35.88 \\
\hline
\end{tabular}

TL: advance time at the end of the area, min; Ea: water application efficiency, \%; Er: water storage efficiency, \% and Vz: infiltrated water volume, $\mathrm{m}^{3}$. 
varies with the increase of $\phi$ and $\theta$. This oscillation confirms the variation which occurs in the water application efficiency with the increase of the values of $\phi$ and $\theta$. It is observed in Table 1 that the lowest values estimated for the water infiltrated volume occur when the values of $\theta$ close to 0.60 and $\phi$ close to 0.51 are considered, while the highest values estimated for the water infiltrated volume happen when values of $\phi$ superior to 0.60 and $\theta$ between 0.51 and 0.61 are considered.

\section{Conclusions}

The simulations of the advance phase by the model SASIS presented discrepancies in the advance time at the end of the area, which did not compromised the prognosis of the balance of water volume, profile of water infiltration or the parameters of the performance of furrow irrigation system with continuous flow.

The analysis of the sensitivity of simulation of the advance phase in the irrigation identified ranges of combinations between spatial and temporal weighted factors of the surface and subsurface flow profiles, which resulted in acceptable discrepancies between simulated and measured advance time.

The model SASIS presented effective mechanisms in the implementation of a number of simulations, in a range of flow between the minimum and maximum allowed values, aiming to determine the relation between flow and water application efficiency, deep percolation and runoff rates and, consequently, to optimize the performance of furrow irrigation system with continuous flow.

\section{References}

[1] F.C. Rezende, M.S. Scalco, E.J. Scaloppi, R. Scardua,
Management alternatives and design in furrow irrigation, in: XVII Brazilian Congress of Agricultural Engineering, Sorocaba, Vol. 1, 1988, pp. 102-114. (in Portuguese)

[2] I.P. Wu, T. Liang, Optimal desing of furrow length of surface irrigation, Journal of the Irrigation and Drainage 96 (1970) 319-332.

[3] J.M. Reddy, W. Clyma, Optimal design of furrow irrigation system, Trans. ASAE 24 (1981) 617-623.

[4] J.L. Merriam, J. Keller, Farm Irrigation System Evaluation: A Guide for Management, 3rd ed. Published by Agricultural and Irrigation Engineering Departament, Utah State University Utah State University, Logan, USA, 1978, p. 271.

[5] M.G. Bos, J. Nugteren, On Irrigation Efficiencies, International Institute for Land Reclamation and Improvement (ILRI), Wageningen, Publication No. 19, The Netherlands, 1974.

[6] E.G. Kruse, D.F. Heermann, Implications of irrigations system efficiencies, Journal of Soil and Water Conservation 32 (1977) 465-470.

[7] T. Strelkoff, N.D. Katopodes, End depth under zero-inertia conditions, Journal of the Hydraulics Division 103 (1977) 599-611.

[8] C.A.V. de Azevedo, Real-time solution of the inverse furrow advance problem, Ph.D. Dissertation, Agricultural and Irrigation Engineering Department, Utah State University, Logon, Utah, USA, 1992, p. 263. (in Portuguese)

[9] C.A.V. de Azevedo, W.R. Walker, Modeling system of checks and control of furrow irrigation, in: XXII Brazilian Congress of Agricultural Engineering, 1993, pp. 2193-2208. (in Portuguese)

[10] W.R. Walker, A.S. Humpherys, Kinematic-wave furrow irrigation model, Journal of the Irrigation and Drainage Division 109 (1983) 377-392.

[11] W.R. Walker, G.V. Skogerboe, Surface Irrigation: Theory and Practice, 1st ed., New Jersey, Prentice-Hall, Inc., Englewood Cliffs, 1987, p. 470.

[12] J.J. Carvalho, C.A.V. Azevedo, J. Henrique, J. Dantas Neto, V.L.A. Lima, R.V. Pordeus, Reflections of spatial and temporal variability of the phase advance and the opportunity time for infiltration of water application time in furrow irrigation for a sandy loam soil, Agricultural Engineering 24 (2004) 455-463. (in Portuguese) 